\title{
Last gasp revisited
}

Edo Bedzra, MD, ${ }^{a}$ Daniel A. Aldea, MS4, ${ }^{b}$ and Gabriel S. Aldea, MD $^{\mathrm{a}}$

From the ${ }^{\mathrm{a} D e p a r t m e n t}$ of Cardiothoracic Surgery, University of Washington, Seattle, Wash; and ${ }^{\mathrm{b}}$ Boston University School of Medicine, Boston, Mass.

Disclosures: Authors have nothing to disclose with regard to commercial support.

Received for publication June 6, 2018; revisions received June 6, 2018; accepted for publication June 7, 2018; available ahead of print Aug 8, 2018.

Address for reprints: Gabriel S. Aldea, MD, Department of Cardiothoracic Surgery, University of Washington, Box 356310, 1959 NE Pacific St, Seattle, WA 98195-6310 (E-mail: aldea@u.washington.edu).

J Thorac Cardiovasc Surg 2018;156:2168-9

$0022-5223 / \$ 36.00$

Copyright (c) 2018 by The American Association for Thoracic Surgery

https://doi.org/10.1016/j.jtcvs.2018.06.032

One hundred years after the introduction of surgical embolectomy (SE) by Trendelenburg and Kirschner, 50 years after the addition of cardiopulmonary bypass to SE by Beall and Cooley, and 2 decades after dramatic evolutions in pharmacologic catheter-based thrombolysis (CBT), acute pulmonary embolus (PE) continues to affect more than 400,000 patients per year in the United States and causes more than 80,000 deaths. ${ }^{1}$ Despite the disease's prevalence and malignancy, there is a paucity of robust clinical data and randomized trials comparing the effectiveness of different strategies. As a result, guidelines rely heavily on helpful but highly selected (biased) clinical experiences and expert opinion. $^{2-4}$

The often dramatic, highly varied acute PE presentations challenge our medical delivery algorithms by requiring rapid, repeated, and accurate multidisciplinary assessments to (1) define anatomic clot burden (size and centrality) and physiologic consequence to differentiate massive from submassive presentations: Massive PE is defined by right ventricular (RV) dysfunction, shock, and organ ischemia, and submassive PE is defined as physiologic RV dysfunction in the absence of shock; (2) rapidly define needs for advanced respiratory and hemodynamic support including mechanical cardiopulmonary support (extracorporeal membrane oxygenation [ECMO]) for those with massive PE; (3) select the best initial and subsequent therapy; (4) reevaluate the effects of the primary intervention; and (5) establish longitudinal follow-up to assess the impact of residual clot burden on the development of and interventions for chronic thromboembolic pulmonary hypertension.

If this task were not challenging enough, acute PE frequently occurs in patients with multiple, unique comorbidities, and thus this dynamic therapeutic decision making also needs to be individualized and contextualized. ${ }^{5-7}$ To date, most guidelines and algorithms are driven by disease burden and evidence of physiologic instability because prior studies demonstrated radiographically quantified clot removal to directly correlate to physiologic improvement in RV function. The success of CBT in contemporary series focused mainly on submassive acute PE

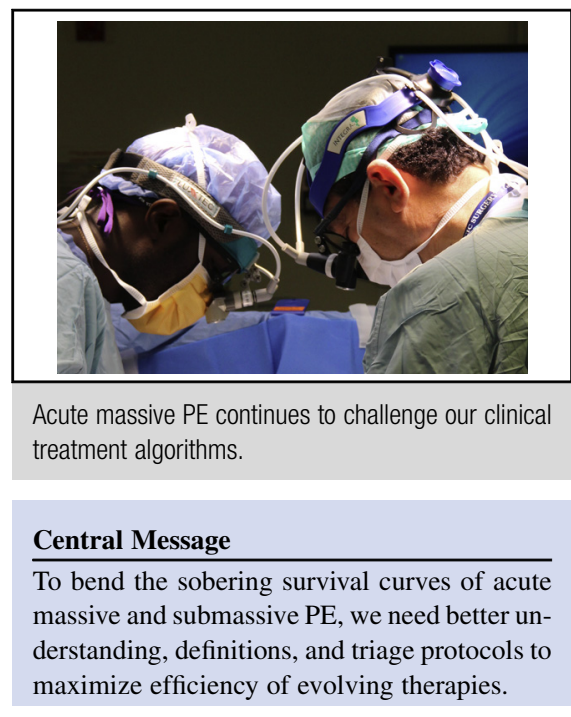

See Article page 2155

presentations and challenges our prior dogmatic thinking that complete or near complete clot extraction is crucial. SEATTLE II and similar studies demonstrated that CBT with ultrasound-guided, local tissue plasminogen activator (tPA) successfully reduced pulmonary artery pressures and anatomic clot burden (computed tomography angiography-based Miller scale) by $30 \%$. This seemingly modest anatomic reduction resulted in dramatic physiologic consequences with a decrease in RV strain and significantly improved survival compared with historical controls treated with systemic lytic therapy, while substantially reducing catastrophic bleeding events (especially intracranial bleeds) associated with systemic lytic therapy. ${ }^{8}$ Furthermore, acute failure of CBT therapy was low $(8 \%)$. It is unclear whether these data can be generalized to patients with massive PE.

Representing the other paradigm is open SE. The aim of this approach is a more extensive (complete or near complete) clot removal with the goal of full and immediate restoration of RV function and, conceptually, the avoidance of chronic sequelae (chronic thromboembolic pulmonary hypertension). The evolution of imaging, surgical expertise, and ECMO technologies challenge the prevailing view that SE should be applied only to patients with massive PE and hemodynamic instability. ${ }^{5-7}$ One could speculate that subsets of high-risk patients with submassive PE also could derive benefit from such intervention when optimally applied. 
Better understanding and revalidation of the impact of current individual treatment options to specific patient subsets are essential for the development of more focused, clinically relevant guidelines.

This important contemporary (1998-2015) meta-analysis by Dr Rajagopal and colleagues describes patient characteristics and outcomes for CBT (41 studies, 1650 patients) and SE (34 studies, 1101 patients) for acute PE. The limitations of such analyses that use pooled retrospective data from institutions with variable expertise and volumes, uncontrolled assignment to specific therapies, inconsistent acute PE classification, unclear cause of acute PE, unknown mode of death, and lack of longer-term follow-up beyond initial hospitalization are obvious. That said, the data are striking and merit a national discussion and engagement on how to better serve this challenging patient population.

The authors conclusively demonstrate that patients undergoing SE versus CBT are sicker, with a higher incidence of massive acute PE and a 5-fold increase in preprocedural cardiopulmonary resuscitation. Preprocedural cardiopulmonary resuscitation carried a high mortality (hazard ratio, 9.8; confidence interval, 5-17), reinforcing prior publications. ${ }^{5-7}$ As expected, after accounting for that variable, there was no statistically significant difference in contemporary mortality for SE and CBT $(6.3 \%$ and $5.6 \%$; hazard ratio, $1.38 ; P=.8$ ). They conclude that each therapeutic approach has a role to play in patients with a particular profile.

We believe the initial clinical presentations of massive $\mathrm{PE}$ and submassive PE should be considered fluid rather than static. Prompt, formal, and frequent reevaluation is required to assess the initial choice of therapy and the efficacy of such therapy. Failure of initial CBT, persistent RV dysfunction, recurrent shock, or organ dysfunction should trigger early reconsideration of venoarterial ECMO support to stabilize the patient and prompt secondary discussions of the timing, risk, and effectiveness of definitive SE. ${ }^{6,7}$

If we hope to bend the sobering survival curves, we need to develop protocols and registries that refine and formalize the promptness and accuracy of initial diagnosis and assessment of physiologic burden based on readily understandable, clinically relevant categories of PE. When identified, effective and prompt triage of higher-risk patients to centers with appropriately staffed and resourced multidisciplinary teams of cardiothoracic surgeons, interventional cardiologists, pulmonologists, radiologists, intensivists, and ethicists is recommended.

\section{References}

1. The Surgeon General's Call to Action to Prevent Deep Vein Thrombosis and Pulmonary Embolism. U.S. Department of Health and Human Services; 2008. Available at: www.ncbi.nlm.nih.gov/books/NBK44178/. Accessed September 21, 2014.

2. Jaff MR, McMurtry MS, Archer SL, Cushman M, Goldenberg N, Goldhaber SZ, et al. Management of massive and submassive pulmonary embolism, iliofemoral deep vein thrombosis, and chronic thromboembolic pulmonary hypertension: a scientific statement from the American Heart Association. Circulation. 2011; 123:1788-830.

3. Konstantinides SV, Torbicki A, Agnelli G, Danchin N, Fitzmaurice D, Galiè N, et al. 2014 ESC guidelines on the diagnosis and management of acute pulmonary embolism. Eur Heart J. 2014;35:3033-69. 3069a-3069k.

4. Kearon C, Akl EA, Comerota AJ, Prandoni P, Bounameaux H, Goldhaber SZ, et al Antithrombotic therapy for VTE disease: antithrombotic therapy and prevention of thrombosis, 9th ed: American College of Chest Physicians evidence-based clinical practice guidelines. Chest. 2012;141(Suppl):e419-94.

5. Kalra R, Bajaj NS, Arora P, Arora G, Crosland WA, McGiffin DC, et al. Surgical embolectomy for acute pulmonary embolism: systematic review and comprehensive meta-analyses. Ann Thorac Surg. 2017;103:982-90.

6. Dudzinski DM, Giri J, Rosenfield K. Interventional treatment of pulmonary embolism. Circ Cardiovasc Interv. 2017;10:e004345.

7. Neely RC, Byrne JG, Gosev I, Cohn LH, Javed Q, Rawn JD, et al. Surgical embolectomy for acute massive and submassive pulmonary embolism in a series of 115 patients. Ann Thorac Surg. 2015;100:1245-52.

8. Piazza G, Hohlfelder B, Jaff MR, Ouriel K, Engelhardt TC, Sterling KM, et al. A prospective, single-arm, multicenter trial of ultrasound-facilitated, catheterdirected, low-dose fibrinolysis for acute massive and submassive pulmonary embolism: the SEATTLE II study. JACC Cardiovasc Interv. 2015;8:1382-92. 